\title{
Punica granatum, lippia sidoides, mikania glomerata e mikania laevegata: ação sobre o biofilme dental
}

Punica granatum, lippia sidoides, mikania glomerata and mikania laevegata: the action on dental biofilme Punica granatum, lippia sidoides, mikania glomerata y mikania laevegata: acción sobre el biofilme dental Rauhan Gomes de QUEIROZ ${ }^{1}$

Sandson Cleyton Ferreira da Silva OLIVEIRA ${ }^{1}$ Lukas Natã Mendes FRAGOSO ${ }^{1}$ Nathalia da Cruz FLORES ${ }^{1}$

Ana Letícia Alcântara FREITAS ${ }^{1}$

Millena Lorrana de Almeida SOUSA ${ }^{1}$

Maria Vitória Calado Ramalho dos SANTOS ${ }^{1}$ Felipe Franklin de JESUS ${ }^{1}$ José Wanderson LACERDA ${ }^{1}$ Layara Maria Vieira LINHARES ${ }^{1}$

Abrahão Alves de OLIVEIRA FILHO ${ }^{2}$

${ }^{1}$ Curso de Graduação em Odontologia da Universidade Federal de Campina Grande, UFCG, Campus de Patos 58708-110, Patos-PB, Brasil ${ }^{2}$ Professor Doutor, Unidade Acadêmica de Ciências Biológicas, Centro de Saúde e Tecnologia Rural, Universidade Federal de Campina Grande UFCG 58428-830 Campina Grande - PB, Brasil

\section{Resumo}

As plantas medicinais vêm sendo mais utilizadas devidos às suas propriedades preventivas, paliativas e curativas, além de ser uma terapia diferente, trazendo inúmeros benefícios aos usuários. A Punica granatum Linn pertence a família Lythracaceae, sendo popularmente conhecida como "Romã". Em forma de colutório a romã pode demonstrar eficácia sobre diversos microorganismos formadores do biofilme dental, além de ajudar na redução do sangramento gengival. A Lippia sidoides Cham pertence à família Verbenaceae, popularmente conhecida como "Alecrim-pimenta". Seu uso tem mostrado uma alta atividade antimicrobiana contra fungos e bactérias, incluindo espécies do gênero Streptococcus mutans, como, também, a redução da gengivite e placa bacteriana. As Mikania glomerata e a Mikania laevegata, pertencem a mesma família Asteraceae e são popularmente conhecidas como "Guaco" e possuem atividade antimicrobiana sobre o Streptococcus mutans. O presente trabalho teve como objetivo realizar uma revisão bibliográfica sobre o uso de fitoterápicos na Odontologia, em especial quanto à atividade microbiana das plantas Punica, Lippia e Mikania, as propriedades farmacológicas dessas e sua atuação sobre o biofilme dental. Trata-se de uma revisão literária do tipo narrativa, onde foi realizada uma seleção de trabalhos científicos já elaborados sobre os aspectos das propriedades farmacoló gicas da Mikania glomerata, Mikana laevigata, Punica granatum Linn. e da Lippia sidoides Cham, através das bases de dados: LILACS, SciELO, MEDLINE, BVS, além de monografias, teses, dissertações e livros. Foi visto na literatura que todas as plantas pesquisadas possuem efeito sobre o biofilme dental quando em baixa concentração e de acordo com suas propriedades químicas e fitoterápicas. Descritores: Odontologia, Fitoterapia, Microbiologia.

\section{Abstract}

Medicinal plants have been used more due to their preventive, palliative and curative properties, besides being a different therapy, bringing innumerable benefits to the users. The Punica granatum Linn belongs to the family Lythracaceae, being popularly known as "Pomegranate". In the form of mouthwash the pomegranate can demonstrate efficacy on several microorganisms forming the dental biofilm, besides helping to reduce gingival bleeding. Lippia sidoides Cham belongs to the family Verbenaceae, popularly known as "Rosemary-pepper". Its use has shown a high antimicrobial activity against fungi and bacteria, including species of the genus Streptococcus mutans, as well as the reduction of gingivitis and plaque. Mikania glomerata and Mikania laevegata, belong to the same family Asteraceae and are popularly known as "Guaco" and have antimicrobial activity on Streptococcus mutans. The objective of this work was to carry out a bibliographic review on the use of phytotherapics in dentistry, especially regarding the microbial activity of the plants Punica, Lippia and Mikania, their pharmacological properties and their performance on the dental biofilm. It is a literary revision of the narrative type, where a selection of scientific works already elaborated on the pharmacological properties aspects of Mikania glomerata, Mikana laevigata, Punica granatum Linn. and Lippia sidoides Cham, through the databases: LILACS, SciELO, MEDLINE, VHL, besides monographs, theses, dissertations and books. It was seen in the literature that all the researched plants have an effect on dental biofilm when in low concentration and according to their chemical and phytotherapeutic properties.

Descriptors: Dentistry, phytotherapy, microbiology.

\section{Resumen}

Las plantas medicinales vienen siendo más utilizadas debido a sus propiedades preventivas, paliativas y curativas, además de ser una terapia diferente, trayendo innumerables beneficios a los usuarios. La Punica granatum Linn pertenece a la familia Lythracaceae, siendo popularmente conocida como "Román". En forma de colutorio a granada puede demostrar eficacia sobre diversos microorganismos formadores del biofilm dental, además de ayudar en la reducción del sangrado gingival. La Lippia serides Cham pertenece a la familia Verbenaceae, popularmente conocida como "Romero-pimienta". Su uso ha mostrado una alta actividad antimicrobiana contra hongos y bacterias, incluyendo especies del género Streptococcus mutans, como también la reducción de la gingivitis y placa bacteriana. Las Mikania glomerata y Mikania laevegata, pertenecen a la misma familia Asteraceae y son popularmente conocidas como "Guaco" y poseen actividad antimicrobiana sobre el Streptococcus mutans. El presente trabajo tuvo como objetivo realizar una revisión de la literatura sobre el uso de fitoterápicos en la Odontología, en especial en cuanto a la actividad microbiana de las plantas Punica, Lippia y Mikania, las propiedades farmacológicas de éstas y su actuación sobre el biopelícula dental. Se trata de una revisión literaria del tipo narrativo, donde se realizó una selección de trabajos científicos ya elaborados sobre los aspectos de las propiedades farmacológicas de la Mikania laevigata, Punica granatum Linn. y de la Lippia serides Cham, a través de las bases de datos: LILACS, SciELO, MEDLINE, BVS, además de monografías, tesis, disertaciones y libros. Se ha visto en la literatura que todas las plantas investigadas tienen efecto sobre el biofilm dental cuando en baja concentración y de acuerdo con sus propiedades químicas y fitoterápicas.

Descriptores: Odontología, Fitoterapia, Microbiología.

O uso de fitoterápicos no Brasil tem um crescimento notório, apesar de ser ainda pouco utilizada pela população. A fitoterapia é uma prática usada há vários séculos e desde então vem sendo aplicada como um recurso terapêutico, auxiliando a medicina na cura e tratamento de patologias. Os fitoterápicos são substâncias obtidas a partir de plantas que podem ser utilizadas como remédios artesanais sob a forma de chás, soluções, comprimidos, 
dentre outros. O uso dos fitoterápicos tem como finalidade prevenir, curar ou minimizar os sintomas das doenças ${ }^{1}$.

Em Odontologia o uso de fitoterápicos ainda é pouco percebido, a inserção de plantas medicinais aos dentifrícios e enxaguantes bucais já é realidade, sendo que diversos extratos de plantas foram testados em estudos científicos com objetivo de avaliar seu potencial de redução da atividade de microrganismos comensais da cavidade bucal. Apesar de a Fitoterapia já ser amplamente utilizada na área médica, sua aplicação na Odontologia ainda é modesta ${ }^{2}$.

Inúmeras substâncias químicas vêm sendo estudadas, com a finalidade de inibir a formação do biofilme dental, desenvolvimento de bactérias, e a adesão de microrganismos à superfície do dente. Em meio a estas substâncias, atualmente se destacam os produtos de origem vegetal por se mostrarem potencialmente ativas no que se trata à sua atividade antimicrobiana sobre diversas espécies de microrganismos ${ }^{3}$.

O biofilme é um aglomerado microbiano composto por células que são imersos numa matriz orgânica de polissacarídeos e adere-se às superfícies duras da cavidade bucal ${ }^{4}$. A cárie dentária e a doença periodontal têm o biofilme como parte de seus fatores etiológicos, ou seja, a remoção física ou química do mesmo auxilia na prevenção daquelas ${ }^{5,6}$.

Diante da vasta quantidade de plantas que podem ser utilizadas para fins terapêuticos na odontologia, o presente estudo teve como objetivo recolher informações através de uma revisão na literatura em artigos e publicações científicas em revistas, sobre o uso fitoterápico de plantas que atuam sobre o biofilme dental.

MATERIAL E MÉTODO

O estudo trata-se de uma revisão literária do tipo narrativa, a qual teve como objetivo reunir o conhecimento científico já elaborado sobre os aspectos das propriedades farmacológicas da Mikania glomerata, Mikana laevigata, Punica granatum Linn. e da Lippia sidoides Cham. As bases de dados utilizadas para a realização deste estudo foram: LILACS (Literatura Latino-Americana e do Caribe em Ciências da Saúde), SciELO (Scientific Electronic Library Online), MEDLINE (Medical Literature Analysis and Retrieval Sistem Online), BVS (Biblioteca Virtual em Saúde), além de monografias, teses, dissertações e livros. Para a busca dos artigos foram utilizadas as palavraschaves em português, inglês e espanhol: Odontologia, Fitoterápicos, Biofilme, Mikana glomerata, Mikana laevigata, Punica granatum, Lippia sidoides.

Os critérios de inclusão foram: pesquisas que abordassem os aspectos das perspectivas do uso fitoterápico da Mikania glomerata Spreng. e Mikania laevigata, Punica granatum Linn. e Lippia sidoides Cham., publicadas em inglês, português ou espanhol, no período de 2004 a 2018, com exceção apenas de artigos clássicos que se apresentaram imprescindíveis para a realização deste estudo. Foram excluídos trabalhos que não apresentassem o conteúdo na íntegra nas bases de dados e bibliotecas pesquisadas; que fossem publicações de anos anteriores; e, com duplicidade (artigos publicados mais de uma vez).

A pesquisa foi realizada nos meses de janeiro a abril de 2018, obtendo-se um total de 857 artigos. Após a análise dos resumos, os 38 trabalhos que atenderam os critérios estabelecidos, foram incorporados para a realização deste estudo. Dessa forma, realizouse a avaliação, agrupamento e síntese das temáticas, expondo o conhecimento produzido sobre o tema proposto.

RESULTADOS E DISCUSSÃO

\section{- Fisiopatologia do biofilme dental}

Define-se biofilme dental como uma comunidade cooperativa, organizada, de células microbianas aderidas a uma superfície úmida e englobado por uma matriz formada por polissacarídeos extracelulares e proteínas microbianas. O biofilme dental constitui assim um microambiente propício ao desenvolvimento bacteriano $^{6,7}$.

Em seu estudo sobre os processos envolvidos na presença da doença cárie, Teixeira et al. ${ }^{8}$ relatam que existem cinco estágios de desenvolvimento do biofilme dental. No primeiro estágio ocorre a adsorção de bactérias na superfície do elemento dentário, tendo como consequência a formação de uma fina película (película adquirida) imediatamente após o surgimento do dente na cavidade oral e, também após a limpeza destes. Posteriormente, as bactérias começam a construir exopolissacarídeos (EPS) insolúveis, sendo que esse processo garante a adesão delas em uma matriz tridimensional, que é o biofilme. A segunda etapa se dá através da interação físico-química das bactérias com o biofilme dentário. Após a adesão com outros microrganismos colonizadores, ocorre a interação entre os receptores específicos de adesão. Em seguida inicia o crescimento populacional dos microrganismos através da 
multiplicação, formando uma superfície tridimensional e funcionalmente organizada. A partir desse ponto temos o biofilme maduro. Por fim, as bactérias podem responder a sinais do meio ambiente e se destacar da superfície, permitindo que colonizem outros locais.

Assim, o biofilme dental se faz importante na saúde do ambiente oral, visto que sua presença em devido equilíbrio protege a cavidade oral contra microrganismos patogênicos devido a interação competitiva entre os microrganismos. Já seu desequilíbrio, pode ocasionar doenças como a cárie onde ocorre um desequilíbrio entre os processos de desmineralização e remineralização dentária ${ }^{9}$.

- Punica granatum Linn. (Romã)

Punica granatum Linn. é o nome científico do fruto conhecido popularmente como Romã, originado da Romãzeira a qual pertence ao reino Plantae, filo Tracheopyta, classe Magnoliopsida, ordem Myrtales, família $L y$ thracaceae ${ }^{10}$. Originado no nordeste da Índia, adapta-se perfeitamente às áreas de climas tropical e subtropical, ou seja, o Nordeste Brasileiro é o lugar ideal para o cultivo dessa planta. $\mathrm{Na}$ cidade de Sousa (PB), há um dos maiores pomares de Romã do país com, aproximadamente, 70 hectares de extensão ${ }^{11,12}$.

A Romãzeira é uma planta que pode medir até 3 metros de altura, de porte arbustivo e apresenta ramificações em formato de espinhos. Suas folhas são pequenas e com consistência de membrana e, suas flores vermelho-alaranjadas encontram-se nas extremidades de seus ramos. $O$ fruto propriamente dito tem aspecto arredondado; casca lisa, brilhante, coloração que varia entre o amarelo e o bordô e algumas manchas escuras; suas sementes são numerosas e dispostas em camadas envolvidas por arilo rosado e um líquido doce ${ }^{11}$.

Da Punica granatum L. podem ser extraídos diversos constituintes químicos, os quais são encontrados em várias partes de seu fruto, como na casca, sementes e em seu suco. Entre seus principais constituintes registrados estão as Antocianinas (Cianidina 3,5diglicosídeo), Flavanóides (quercetina), Ácidos Fenólicos (Ácido Caféico) e Taninos ${ }^{13}$.

Os estudos realizados com a Punica granatum Linn tem demonstrado significativa eficácia sobre diversos microrganismos, como demonstra o estudo de Dabholkar et al. ${ }^{14}$, cujo objeto foi avaliar o efeito do enxaguatório bucal a base de romã sobre bactérias formadoras de biofilme. Os resultados obtidos evidenciam a eficácia da planta sobre a inibição de microrganismos bucais, porém em diferentes concentrações, sendo possível utilizar esse tipo de enxaguante em longo prazo, em substituição de outros a base agentes químicos.

Para avaliar a efetividade da $P$. granatum sobre o biofilme dental e a inflamação da gengiva, Silva ${ }^{15}$ usou a planta como base de um enxaguante bucal. Em sua pesquisa o autor utilizou um enxaguante a base de Romã e outro a base de clorexidina. Os achados mostraram que apesar da clorexidina apresentar maior efetividade sobre o biofilme dental quando comparado a $P$. granatum, ambos demonstraram a mesma eficácia para redução do sangramento gengival. Assim pode-se concluir que o enxaguatório de romã pode ser utilizado como fitoterápico para o controle do biofilme dental e a inflamação gengival.

Em sua revisão bibliográfica acerca do uso da $P$. granatum, Werkman et al. ${ }^{16}$, buscou estudos sobre a aplicabilidade do vegetal como antifúngico, antibacteriano, antioxidante, antineoplásico, seu uso na odontologia, dentre outros. Assim, puderam concluir, a partir dos achados literários, que a planta tem um grande potencial sobre patógenos, em especial suas propriedades antiinflamatória e antimicrobiana, sendo assim podendo ser utilizada na área odontológica e médica.

Por tanto, estudos com a finalidade de avaliar e conhecer a efetividade da Punica granatum em suas propriedades antifúngica, antimicrobiana e antiinflamatória, apresentam resultados positivos, sendo possível utilizar a planta de diferentes formas (chás, bochechos) enquanto fitoterápico para a área da saúde s0,17-20. $^{10}$.

\section{- Lippia sidoides Cham. (Alecrim-pimenta)}

A Lippia sidoides Cham. é uma planta usualmente conhecida como "alecrim-pimenta", podendo também ser chamada pelos nomes de "alecrim-do-nordeste" e "estrepa-cavalo". Ela pertence à família verbenaceae, tem característica arbustiva, seu tamanho pode variar de 2 a $3 \mathrm{~m}$ de altura e é nativa de regiões semi-áridas. No Brasil, ela pode ser encontrada facilmente nos estados do Rio Grande do Norte, Ceará e na região norte do estado de Minas Gerais $^{21-26}$.

O óleo essencial obtido da planta alecrim-pimenta é composto principalmente por mono e sesquiterpenos, no qual o Timol (56,7\%$59,65 \%$ é destacado como principal constituinte. Além dele, tem-se o carvacrol (16,7\%-19\%) e outras substâncias, tais como felandreno, cariofileno $(1,1 \%-10,6 \%), p$-cimeno $(7,1 \%-9,08 \%)$ e mirceno $(0,86 \%-5,46 \%)$. A composição pode variar dependendo da região geográfica de coleta, da variedade, da idade da 
planta, bem como também dos métodos empregados na drenagem e extração do óleo. Este, por sua vez, tem grande valor comercial e farmacológico, devido aos constituintes encontrados nas folhas, que possuem atividade inseticida, fungicida, bactericida, leishimanicida, larvicida, acaricida, anti-inflamatória e antiespasmódica ${ }^{21,25,27,28}$.

A atividade antimicrobiana dos óleos essenciais está associada à sua característica lipolítica, causando acúmulo na membrana celular e lesões associadas à depleção de energia ${ }^{29}$.

$\mathrm{Na}$ Medicina, seu uso está comumente vinculado na forma de infusões e inalações, rinites alérgicas, e no tratamento de vagina, boca e infecções da garganta. Já na Odontologia, estudos prévios dos componentes da planta têm mostrado uma potente atividade antimicrobiana contra fungos e bactérias, incluindo espécies do gênero Streptococcus mutans e a redução severa de gengivite, placa bacteriana e infiltrado inflamatório em cães ${ }^{21}$.

Por fim, com a finalidade de se obter um espectro de ação do alecrim-pimenta, estudos na área farmacológica foram realizados reparando que a Lippia sidoides não apresentava toxicidade em suas doses estudadas e mostrava-se como um agente antiinflamatório gastroprotetor ${ }^{30}$.

\section{- Mikania Glomerata e Mikania Laevegata} (Guaco)

A Mikania glomerata e a Mikania laevigata são espécies pertence à família Asteraceae, e geralmente são conhecidas como "Guaco". O Guaco é uma das plantas medicinais brasileiras mais consumidas e é receitado desde a antiguidade pela medicina popular devido a sua atividade antiinflamatória ${ }^{31,32}$. Embora as espécies possuam constituição química semelhante, elas apresentam características físicas diferentes ${ }^{33}$.

A Mikania glomerata é uma espécie de subarbusto silvestre de crescimento escandente, com folhagem densa e perene que possui um caule cilíndrico, ramificado, glabro. A planta apresenta coloração verde-claro quando jovem e passa a ser arroxeada e cinzentoescura nas partes suberificadas. Por outro lado, a Mikania laevigata é uma espécie também de porte subarbustivo, mas com hábito de trepadora volúvel, seu caule é cilíndrico, lenhoso, e de coloração castanha acinzentada nas partes mais velhas e verde clara nas partes próximas às pontas. É uma planta de clima subtropical, quente e úmido, devendo-se evitar regiões de clima frio, podendo ser cultivada a pleno sol ou em sombreamento parcial ${ }^{34}$.
Bolina et al. $^{35}$ realizaram uma prospecção fitoquímica com o intuito de avaliar e conhecer a composição química das duas espécies, e detectaram a presença de cumarina com percentual semelhante em ambas, o que permite que as espécies sejam usadas de maneira indistinta. Além disso, apresentam outros compostos como os triterpenos, esteróides e heterosídeos flavônicos.

$\mathrm{Na}$ composição química de Mikania glomerata podemos encontrar ainda alguns óleos essenciais, entre eles sesquiterpenos e diterpenos do tipo caurano, e.g. ácidos caurenoico, grandiflórico, cinamoilgrandiflórico e caurenol. E metabólitos secundários como sitosterol, friedelina, estigmasterol, taninos hidrolisáveis, flavonoides e saponina. É válido ressaltar que os metabólitos secundários variam em proporção de acordo com vários fatores como, por exemplo, radiação UV, Temperatura, disponibilidade de nutrientes e água no sol, e entre outros ${ }^{36}$.

Quanto à ação antimicrobiana, é possível encontrar na literatura que ambas as espécies são consideradas antibacteriana, anticândida e antiprotozoária, quanto a ação sobre o biofilme dental, estudos mais recentes, mostram que a Mikania apresenta atividade antimicrobiana sobre S. mutans ${ }^{37}$.

$\mathrm{Na}$ Medicina, o tratamento com a Mikania glomerata é indicado em casos de pneumoconiose, que é caracterizada por inflamação pulmonar causada pela exposição ao pó de carvão. A utilização reduz a infiltração pulmonar inflamatória induzida pelo pó de carvão, o que aponta o fitoterápico como preventivo de lesão pulmonar oxidativa ${ }^{36}$.

$\mathrm{Na}$ Odontologia, estudos apontam que sua ação antimicrobiana sobre o biofilme se deve aos ácidos cupressênico e diterpênico da Mikania que, atuando sobre o esse em baixa concentração, demonstram serem promissores agentes anticárie e antiplaca ${ }^{38}$.

CONCLUSÃO

Com base nos resultados obtidos observa-se que várias pesquisas tem apontado o efeito antimicrobiano de compostos obtidos através da utilização da Punica granatum Linn, Lippia sidoides Cham e da Mikania glomerata. 0 emprego desses compostos na odontologia pode ser considerado como um apoio na terapia de diversos fatores etiológicos que desencadeiam a doença cárie e a doença periodontal, como, por exemplo, a inibição de microrganismos bucais, incluindo a espécie do gênero Streptococcus mutans, assim como, para ação de redução do sangramento gengival. 


\section{REFERÊNCIAS}

1. Francisco KSF. Fitoterapia: uma opção para o tratamento odontológico. Rev Saúde.2010;4(1): 18-24.

2. de Melo Aleluia, C, Procópio VC, Oliveira MTG, Furtado PGS, Giovanni JFG, Mendonça, SMS. Fitoterápicos na Odontologia. Rev odontol. UNICID. 2017;27(2):126-34.

3. Portela GS. Bioprospecção de plantas medicinais com atividade antimicrobiana e antiquorum sensing. João Pessoa [dissertação]. João Pessoa: Faculdade de Odontologia da Universidade Federal da Paraíba (UFPB); 2011.

4. Uppuluri P, Dinakaran H, Thomas DP, Chaturvedi AK, Lopez-Ribot JL. Characteristics of Candida albicans biofilms grown in a synthetic urine medium. J Clin Microbiol, 2009; 47(12):4078-83.

5. Eto FS, Raslan AS, Cortelli JR. Características microbianas na saúde e doença periodontal. Rev biocienc. 2003;9(2):45-51.

6. Tavares AMB. Produtos de origem vegetal: uma nova estratégia na prevenção da cárie dentária [dissertação]. Coimbra: Faculdade de Farmácia da Universidade de Coimbra; 2016.

7. Salli KM, Ouwehand AC. The use of in vitro model systems to study dental biofilms associated with caries: a short review. J Oral Microbiol. 2015;7:26149.

8. Teixeira KIR, Bueno AC, Cortês ME. Processos físico-químicos no biofilme dentário relacionados a produção da cárie. Quim nova esc. 2010;32(3):145-50.

9. Venâncio GN, Dária PC, Souza MS. Cárie dentária em indivíduos entre 35 e 44 anos do município de Manaus-AM: análise dos dados do Projeto SB Brasil 2010. Arch Health Invest. 2017;6(3):115-19.

10.de Oliveira LML. Punica granatum: quantificação de polifenóis de extratos e potencial antifúngico contra Candida albicans. São Paulo [tese]. Araçatuba: Faculdade de Odontologia de Araçatuba - UNESP; 2016.

11. Batista JFR. Potencial antibacteriano da Punica granatum Linn. (Romã) na Odontologia: Revisão de literatura. Campina Grande [monografia] Campina Grande: Faculdade de Odontologia da Universidade Estadual da Paraíba - UEPB; 2014.

12. dos Santos Moreira I, Rocha RHC, Paiva EP, Silva HS, Sousa FA. Biometria e componentes físico-químicos de romã armazenada sob refrigeração. Pesq Agropec Trop. 2015;45(2): 209-15.

13. Nascimento Júnior BJ, Santos AMT, Souza AT, Santos EO, Xavier MR, Mendes RL et al. Estudo da ação da romã (Punica granatum L.) na cicatrização de úlceras induzidas por queimadura em dorso de língua de ratos Wistar
(Rattus norvegicus). Rev bras plantas med. 2016;18(2):423-32.

14.Dabholkar CS, Shah M, Kathariya R, Bajaj M, Doshi Y. Comparative evaluation of antimicrobial activity of pomegranate-containing mouthwash against oral-biofilm forming organisms: an in vitro microbial study. J Clin Diagn Res. 2016;10(3):65-9.

15. Silva FRS. Avaliação clínica da efetividade de um enxaguatório (Punica granatum Linn.) sobre o controle de biofilme dentário e inflamação gengival em escolares. Campina Grande [monografia]. Campina Grande: Faculdade de Odontologia da Universidade Estadual da Paraíba - UEPB; 2014.

16. Werkman C, Granato DC, Kerbauy WD, Sampaio FC, Brandão AAH, Rode SM. Aplicações terapêuticas da Punica granatum L.(romã). Rev bras plantas med. 2008; 10(3):104-11.

17. Schreiner F, Retzlaff G, Siqueira MFR, Rezende EC, Simão LC, Kozlowski-Junior VA et al. Uso do chá de Punica granatum (romã) no controle da aderência de bactérias orais em ligaduras ortodônticas. ROBRAC. 2009;18(45): 56-61.

18. Endo EH, Ueda-Nakamura T, Nakamura CV, Filho BP. Activity of spray-dried microparticles containing pomegranate peel extract against Candida albicans. Molecules. 2012;17(9): 10094-107.

19. Oh SK, Chang HJ, Chun HS. Pomegranate (Punica granatum L.) Peel Extract Inhibits Quorum Sensing and Biofilm Formation Potential in Yersinia enterocolitica. Microbiol Biotechnol. Lett.2015;43(4):357-66.

20. Sivakumar T, Sukumar S, Yogananth $N$. Isolation and Identification of Dental caries causing Actinomycetes and antimicrobial activity of Punica granatum. Int $\mathrm{J}$ Curr Res Biol Med. 2016;1(4):1-14.

21. Rodrigues ISC, Tavares VN, Pereira SLS, Costa FN. Antiplaque and antigingivitis effect of Lippia Sidoides: a double-blind clinical study in humans. J Appl Oral Sci. 2009;17(5):404-7.

22. de Morais SR, Oliveira TL, Bara MT, da Conceição EC, Rezende $\mathrm{MH}$, Ferri $\mathrm{PH}$, de Paula JR. Chemical Constituents of Essential Oil from Lippia sidoides Cham. (Verbenaceae) Leaves Cultivated in Hidrolândia, Goiás, Brazil. Int J Anal Chem. 2012;2012:363919.

23. Veras HN, Rodrigues FF, Colares AV, Menezes IR, Coutinho HD, Botelho MA et al. Synergistic antibiotic activity of volatile compounds from the essential oil of Lippia sidoides and thymol. Fitoterapia. 2012;83(3):508-12.

24. Veras HN, Rodrigues FF, Botelho MA, Menezes IR, Coutinho HD, da Costa JG. Antimicrobial effect of Lippia sidoides and thymol on Enterococcus faecalis biofilm of the 
bacterium isolated from root canals. ScientificWorldJournal. 2014;2014:471580.

25. Da Silva FP, Aires PA, Praxedes Neto RAL, Aguiar WF, Costa FN, Viana FAC, et al. Avaliação dos extratos de anacardium occidentale linn e lippia sidoides cham no processo de cicatrização tecidual. Estudo histológico em dorso de ratos. Braz J Periodontol. 2013;23(4):18-25.

26. de Albuquerque ACL, Pereira MSV, Pereira JV, Costa MRMI. Efeito antimicrobiano do extrato da lippia sidoides Cham. sobre microrganismos cariogênicos. Arq odontol. 2016;44(4):5-10.

27. Carvalho Júnior WGO, Melo MTP, Martins ER. Fenologia do alecrim-pimenta (Lippia sidoides Cham.) em área de Cerrado, no norte de Minas Gerais, Brasil. Rev bras plantas med. 2011; 13(2):223-29.

28. Costa OS, Souza EB, Brito EHS, Fontenelle ROS. Antimicrobial activity and therapeutic potential of the genus Lippia sensu lato (Verbenaceae). Hoehnea. 2017;44(2):158-71.

29. Castro CE, Ribeiro JM, Diniz TT, Almeida AC, Ferreira LC, Martins ER, et al. Antimicrobial activity of Lippia sidoides Cham.(Verbenaceae) essential oil against Staphylococcus aureus and Escherichia coli. Rev bras plantas med. 2011;13(3):293-97.

30. Pereira MSV, Pereira JV, Albuquerque ACL, Araújo CRF, Diniz DN, Macêdo-Costa MR et al. Plantas medicinais na odontologia: potencial anti microbiano. João Pessoa: EDUFPB; 2010.

31. Santana LCLR, Brito MRM, Oliveira GLS, Citó AMGL, Alves CQ, David JP et al. Mikania glomerata: Phytochemical, pharmacological, and neurochemical study.J Evid Based Complementary Altern Med. 2014;2014: 1-11.

32. Moreira MR, Souza AB, Soares S, Bianchi TC, de Souxa Eugênio, Lemes DC et al. entKaurenoic acid-rich extract from Mikania glomerata: In vitro activity against bacteria responsible for dental caries. Fitoterapia. 2016; 112:211-6.

33. [33] Laurella LC, Cerny N, Bivona AE, Sánchez Alberti A, Giberti G, Malchiodi EL, et al. Assessment of sesquiterpene lactones isolated from Mikania plants species for their potential efficacy against Trypanosoma cruzi and Leishmania sp. PLoS Negl Trop Dis.2017; 11(9):e0005929.

34. Pereira CS. Estudo comparativo da atividade anti-inflamatória dos extratos aquosos de mikania glomerata (sprengel) e mikania laevigata (schultz bip ex Baker) [dissertação]. Campinas: Faculdade de Ciências Médicas da Universidade Estadual de Campinas Unicamp; 2016.

35. Bolina RC, Garcia EF, Duarte MG. Estudo comparativo da composição química das espécies vegetais Mikania glomerata Sprengel e Mikania laevigata Schultz Bip. ex Bake. Rev bras farmacogn. 2009;19:294-98.

36. Czelusniak KE, Brocco A, Pereira DF, Freitas GBL. Farmacobotânica, fitoquímica e farmacologia do Guaco: revisão considerando Mikania glomerata Sprengel e Mikania laevigata Schulyz Bip. ex Baker. Rev bras plantas med. 2012;14(2):400-9.

37. Yatsuda R, Rosalen PL, Cury JA, Murata RM, Rehder VL, Melo LV, et al. Effects of Mikania genus plants on growth and cell adherence of mutans streptococci. J Ethnopharmacol. 2005; 97(2):183-89.

38. Yatsuda R. Efeito antimicrobiano in vitro da Mikania laevigata e da Mikania glomerata sobre estreptococos do grupo mutans. Piracicaba [dissertação]. Piracicaba: Faculdade de Odontologia de Piracicaba da Universidade Estadual de Campinas - Unicamp; 2004.

\section{CONFLITO DE INTERESSES}

Os autores declaram não haver conflitos de interesse

\section{AUTOR PARA CORRESPONDÊNCIA}

\author{
Abrahão Alves de Oliveira Filho \\ Unidade Acadêmica de Ciências Biológicas do Centro \\ de Saúde e Tecnologia Rural da Universidade Federal \\ de Campina Grande \\ 58428-830 Campina Grande - PB, Brasil \\ E-mail: abrahao.farm@gmail.com
}

Submetido em 13/05/2019 Aceito em 28/08/2020 